\title{
Smear-positive pulmonary tuberculosis patients with previously abnormal chest radiographs: missed opportunities for early diagnosis
}

\author{
Lovel Corpuz Galamay ${ }^{1}, \mathrm{MD}$, Cynthia Bin Eng $\underline{\text { Chee }}^{1,2}$, MBBS, FRCP, Kyi Win $\underline{K h i n ~ M a r}^{1}$, MBBS, MMed, Bih Qin Lau ${ }^{3}$, MD,
} Yee Tang Wang $^{1,2}$, MBBS, FRCP

INTRODUCTION It is vital to diagnose and treat pulmonary tuberculosis (PTB) early, in the interests of the patient and public health. At the Singapore Tuberculosis Control Unit (TBCU), it was not uncommon to discover abnormal chest radiographs (CXRs) predating their PTB diagnosis by months to years in the electronic medical records (EMRs) of sputum acid-fast bacilli smear-positive patients. Our study explored this observation.

METHODS The EMRs of sputum smear-positive PTB patients treated at the TBCU from January to July 2014 were viewed for abnormal CXRs preceding their PTB diagnosis. Information in the EMRs pertaining to the indication for the CXRs, radiological reports (including whether the possibility of PTB was stated) and action taken was captured.

RESULTS Of the 254 sputum smear-positive patients, 108 had previous CXRs in their EMRs, of whom 39 (36.1\%) had previous CXRs compatible with PTB. Most of these were performed in tertiary institutions and for reasons unrelated to PTB. No action was taken in response to these CXRs in 24 (61.5\%) patients. 27 (69.2\%) patients had abnormal CXRs dating back more than six months, with 12 (30.8\%) dating back more than 30 months before the PTB diagnosis. Patients aged $\geq 55$ years were significantly more likely to have previous CXRs that were compatible with PTB.

CONCLUSION PTB patients may be asymptomatic or have chronic indolent disease and remain undiagnosed for years. There is a need for vigilance within our healthcare system to seize opportunities for early diagnosis and treatment to prevent the spread of this infectious disease in Singapore.

Keywords: acid-fast bacilli, chest radiograph, delayed diagnosis, pulmonary tuberculosis

\section{INTRODUCTION}

The World Health Organization (WHO) declared tuberculosis (TB) a global health emergency in 1993. 25 years later, the WHO estimates that there were 1.6 million deaths and ten million new TB cases in 2017, with eight countries (India, China, Indonesia, the Philippines, Pakistan, Nigeria, Bangladesh and South Africa) accounting for $66 \%$ of these cases. ${ }^{(1)}$

Singapore's TB rate has stagnated at about 40 per 100,000 population since $2008 .^{(2)}$ The preceding decade had seen the country's TB rate decline from 58 per 100,000 population in 1998 to 35 per 100,000 population in 2007 due to the initiatives of the Singapore TB Elimination Programme (STEP) launched in 1997. ${ }^{(3,4)}$ We believe that the decade-long stagnation of our TB rate is due to changes in population demographics (i.e. the rapidly ageing population, influx of immigrants and migrant workers from high-TB-burden countries) over the last 15 years and ongoing transmission of TB in a densely populated, urbanised setting. To mitigate transmission, it is vital to detect and treat every TB case as early as possible. At the TB Control Unit (TBCU), the national referral centre where $70 \%$ of the country's TB cases are treated, we observed that a worrying number of sputum acid-fast bacilli (AFB) smear-positive pulmonary TB (PTB) patients had previous abnormal chest radiography (CXRs) in their electronic medical records (EMRs) that had not triggered investigations such as sputum AFB smear or TB molecular testing and mycobacterial cultures, which would have led to diagnosis and treatment of this infectious disease. We conducted the present study to investigate this observation.

\section{METHODS}

Ethics approval was obtained from the NHG Domain Specific Review Board (reference no. 2015/00058) for this retrospective study. All newly diagnosed, sputum AFB smear-positive PTB patients seen in the TBCU from January to July 2014 were identified from the STEP Notification Registry. We reviewed the medical records of the 254 notified cases using the patients' paper case files and EMRs. Any CXRs preceding the last abnormal CXR and their findings were noted. Information regarding the centre where these CXRs were done, the indication for the CXR, whether the possibility of TB was stated in the radiological report, and actions (if any) taken for the abnormal CXR was collected. Abnormal CXRs in the EMR preceding the PTB diagnosis were compared with the CXR used by the TBCU consultant to make the PTB diagnosis to determine PTB compatibility and disease progression. The time interval from the first abnormal CXR to PTB diagnosis (taken as date of TB notification) was ascertained.

Data analysis was done using SPSS Statistics version 17.0 (SPSS Inc, Chicago, IL, USA). Cases without a previous CXR prior

${ }^{1}$ Tuberculosis Control Unit, ${ }^{2}$ Department of Respiratory and Critical Care Medicine, Tan Tock Seng Hospital, ${ }^{3}$ Ministry of Health Holdings, Singapore Correspondence: Prof Wang Yee Tang, Emeritus Consultant, Tuberculosis Control Unit, Tan Tock Seng Hospital, 144 Moulmein Road, Singapore 308089. yee_tang_wang@ttsh.com.sg 


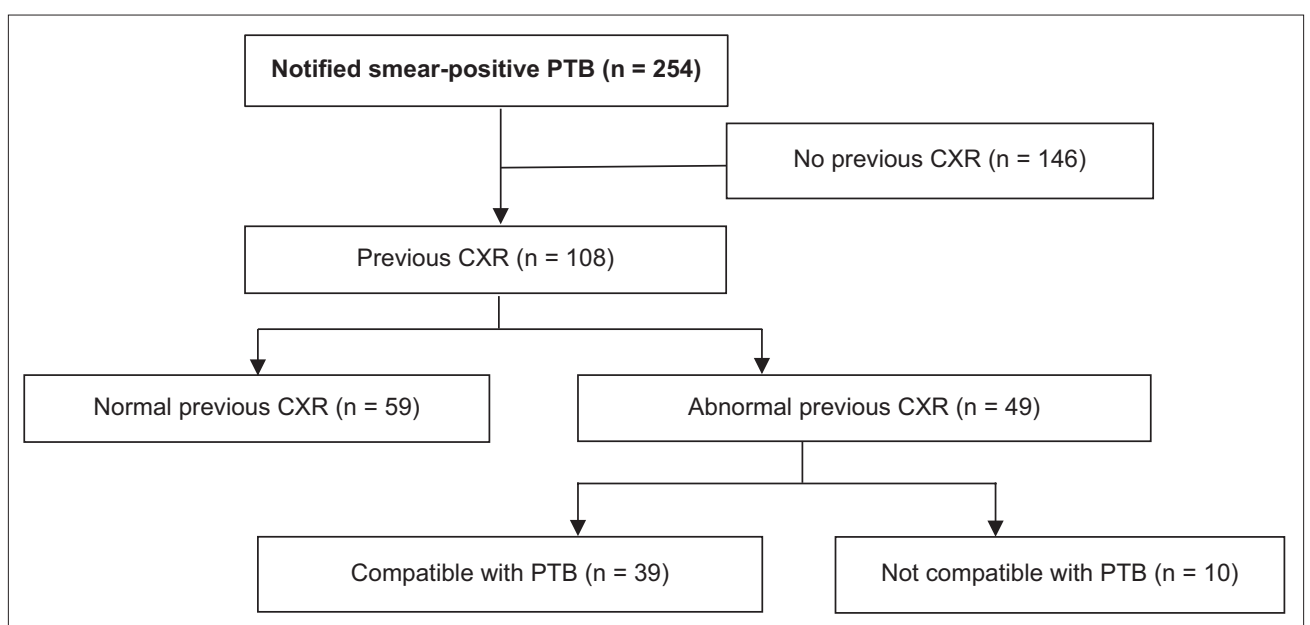

Fig. 1 Flowchart shows the process of data collection. CXR: chest radiograph; PTB: pulmonary tuberculosis

to PTB diagnosis were excluded from the analysis. Descriptive analysis was done for cases with previous abnormal CXRs compatible with PTB. Pearson's chi-square test was used to compare the background characteristics of those with a previous normal CXR and those with a CXR that was compatible with PTB. A p-value $<0.05$ was considered to be statistically significant.

\section{RESULTS}

Of the 254 sputum AFB smear-positive PTB cases treated at the TBCU during the study period, 108 (42.5\%) patients had previous CXRs in their EMR: 49 (45.4\%) had abnormal CXRs, of whom 39 (36.1\%) out of 108 patients had CXRs compatible with PTB (Fig. 1). Of these, 35 (89.7\%) showed disease progression since the previous CXR and 21 (53.8\%) had more than one abnormal CXR predating the TB diagnosis.

The time intervals between the first previously abnormal CXR and the TB diagnosis (taken as date of TB notification) were noted (Fig. 2). The median and mean durations between the first available abnormal CXR and the PTB diagnosis were 14 months and 24 months, respectively (range 1.2-107.6 months). Abnormal CXRs dated back more than six months before PTB was diagnosed in $27(69.2 \%)$ patients, more than 12 months in 21 (53.8\%), and more than 30 months in 12 (30.8\%) patients.

Patients who were aged 55 years and above were significantly more likely to have previous CXRs compatible with PTB (Table I). Gender, ethnicity, country of birth, smoking status, degree of sputum AFB smear positivity, the centre where CXR was done and comorbidities (e.g. diabetes mellitus, cancer and end-stage renal failure) were not associated with increased likelihood of having a previous abnormal CXR. None of the patients in the two groups were HIV-infected.

The majority ( $n=32,82.1 \%$ ) of the 39 patients with previous abnormal CXRs had them performed in tertiary institutions: emergency departments $(n=16)$; cardiology departments $(n=4)$; general medicine departments $(n=2)$; and at respiratory medicine $(n=1)$, obstetrics and gynaecology $(n=1)$, infectious disease $(n=1)$, general surgery $(n=2)$ and neurology departments $(n=1)$. Information pertaining to institution and department was not available for four patients.

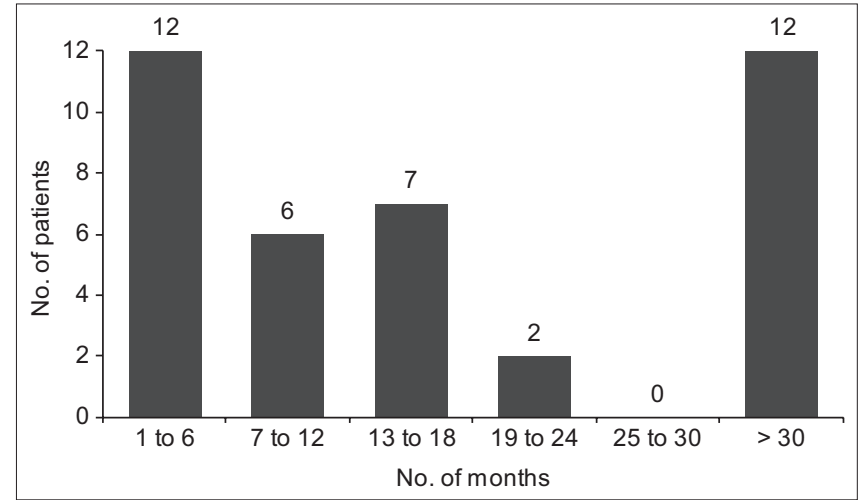

Fig. 2 Chart shows the time interval between the first previously abnormal radiograph and the diagnosis of tuberculosis (taken as the date of notification).

Among the 30 patients in whom the indication for CXR could be ascertained, the majority $(n=17)$ had CXRs performed for reasons unrelated to PTB. These reasons were falls $(n=4)$, road traffic accidents $(n=2)$, physical assault $(n=1)$, preoperative screening $(n=2)$, cerebrovascular accident $(n=1)$, biliary colic $(n=1)$, leg swelling due to proteinuria $(n=1)$, asteatotic eczema $(n=1)$, leg cellulitis ( $n=1)$, aortic dissection $(n=1)$, cholelithiasis $(n=1)$ and abnormal chest findings of basal crepitations on a background of ovarian carcinoma $(n=1)$. The remaining 13 patients had CXRs for evaluation of symptoms possibly attributable to PTB (i.e. cough, fever, chest pain). In these patients, the mean and median durations between the first abnormal CXR and the PTB diagnosis were 14 months and nine months, respectively, whereas they were 23 months and 14 months, respectively, among the 17 patients with CXRs performed for reasons unrelated to PTB.

The eventual diagnosis of smear-positive PTB was made in 27 patients when they were admitted with PTB symptoms, diagnosed with PTB and referred to the TBCU for management upon discharge, and in 12 patients when they were referred as outpatients for PTB symptoms to the TBCU (five from polyclinics, three from emergency departments, three from specialist respiratory clinics and one from a general practitioner).

In the CXR reports of the 39 patients with abnormal CXRs, descriptions compatible with PTB (i.e. 'scarring', 'granuloma', 
Table I. Comparison of background characteristics between patients with previous normal CXR and those with previous CXR compatible with PTB.

\begin{tabular}{|c|c|c|c|}
\hline \multirow[t]{2}{*}{ Characteristic } & \multicolumn{2}{|c|}{ No. (\%) } & \multirow[t]{2}{*}{ p-value } \\
\hline & $\begin{array}{l}\text { Normal } \\
(n=59)\end{array}$ & $\begin{array}{l}\text { Abnormal } \\
(n=39)\end{array}$ & \\
\hline Age (yr) & & & 0.043 \\
\hline$<55$ & $35(59.3)$ & $15(38.5)$ & \\
\hline$\geq 55$ & $24(40.7)$ & 24 (61.5) & \\
\hline Gender & & & 0.981 \\
\hline Female & $15(25.4)$ & $10(25.6)$ & \\
\hline Male & $44(74.6)$ & $29(74.4)$ & \\
\hline Ethnicity & & & 0.576 \\
\hline Chinese & $36(61.0)$ & $21(53.8)$ & \\
\hline Malay & $15(25.4)$ & $14(35.9)$ & \\
\hline Indian & $6(10.2)$ & $2(5.1)$ & \\
\hline Others & $2(3.4)$ & $2(5.1)$ & \\
\hline Country of birth & & & 0.184 \\
\hline Singapore-born & $49(83.1)$ & $28(71.8)$ & \\
\hline Foreign-born & $10(16.9)$ & $11(28.2)$ & \\
\hline Smoking & & & 0.766 \\
\hline No & $26(44.1)$ & $16(41.0)$ & \\
\hline Yes & $33(55.9)$ & $23(59.0)$ & \\
\hline Smear & & & 0.484 \\
\hline $1+$ and $2+$ & $33(55.9)$ & $19(48.7)$ & \\
\hline $3+$ and $3+$ & $26(44.1)$ & $20(51.3)$ & \\
\hline Centre* & & & 0.830 \\
\hline Institution ( $n=42)$ & $37(88.1)$ & $32(86.5)$ & \\
\hline Non-institution $(n=37)$ & $5(11.9)$ & $5(13.5)$ & \\
\hline Comorbidities & & & 0.903 \\
\hline No & $28(47.5)$ & $19(48.7)$ & \\
\hline Yes & $31(52.5)$ & $20(51.3)$ & \\
\hline
\end{tabular}

*Percentages are calculated based on available data.

'cavity', 'fibrosis') were used in 14 reports, while 'opacities' and 'consolidation' were used in 29 reports. 'Tuberculosis' was mentioned in four CXR reports. More than one description could be used in each report (Fig. 3). No action was taken in response to the previous abnormal CXRs in 24 (61.5\%) of the 39 cases (Fig. 4). For the remaining 15 patients, five were prescribed antibiotics, six were referred to respiratory physicians for evaluation, and four were admitted for reasons unrelated to the abnormal CXRs. During the admission, the abnormal CXRs were noted and computed tomography (CT) of the thorax was performed as inpatients; PTB was mentioned in three of the CT thorax reports. Despite the mention of suspicion of PTB in the four CXR and three CT thorax reports (all in different patients), no sputum tests for TB were performed in response to these reports.

\section{DISCUSSION}

We found that $36.1 \%(n=39)$ of sputum AFB smear-positive PTB patients with previous CXRs available in their EMRs had prior radiological evidence of active PTB. Most of these CXRs

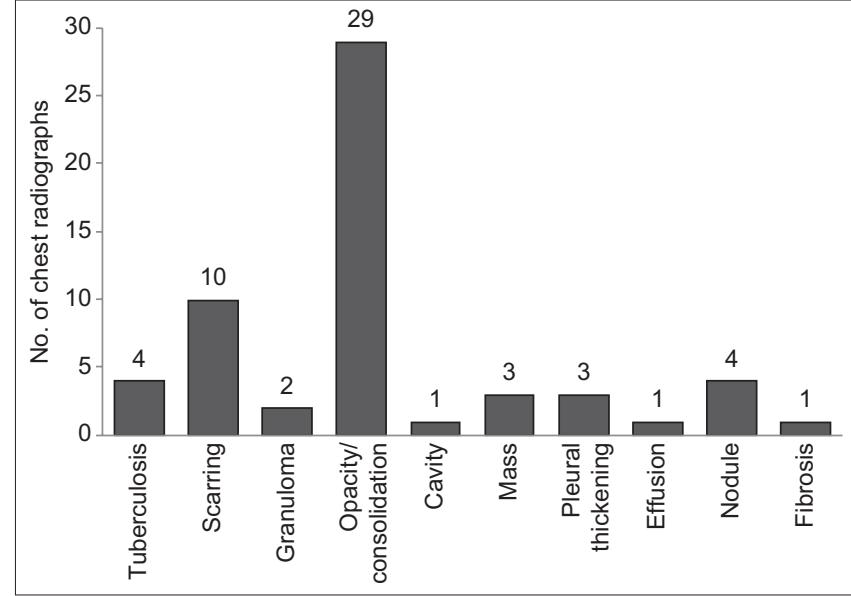

Fig. 3 Chart shows the descriptions used by radiologists in the reports of the 39 patients with abnormal radiographs. Each report could have more than one description.

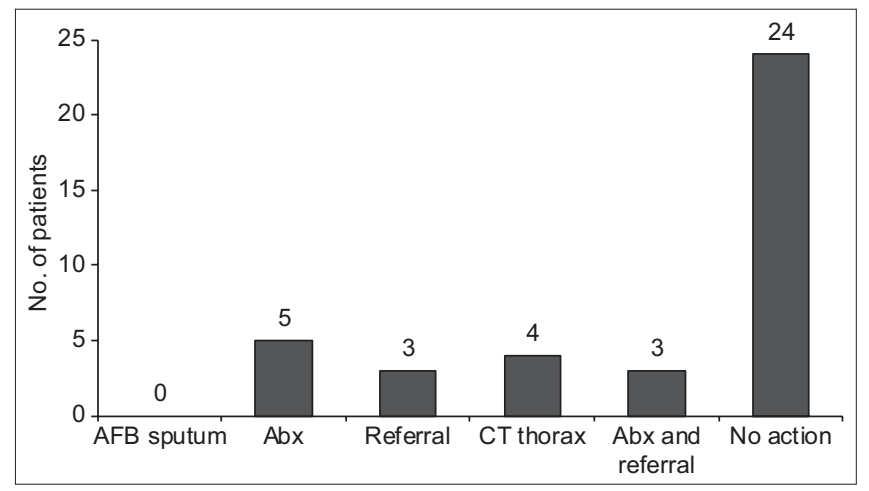

Fig. 4 Chart shows the actions taken in response to the previous abnormal radiographs. Abx: antibiotics; AFB: acid-fast bacilli; CT: computed tomography

were performed in tertiary institutions for reasons unrelated to TB. No action was taken in response to the abnormal CXRs in $24(61.5 \%)$ patients. The majority of the abnormal CXRs dated back more than six months before the diagnosis of smear-positive PTB. Previous CXRs compatible with PTB were more likely to be found in patients aged 55 years and above.

Active TB mostly affects the lungs and has a wide spectrum of manifestation, ranging from subclinical to fulminant, fatal disease. ${ }^{(5)}$ It may run a chronic indolent course, and patients with culture-positive PTB have even been known to be asymptomatic. This is demonstrated in our patients who had evidence of PTB on CXRs that were performed for unrelated reasons dating back months to years before they were diagnosed with smear-positive PTB. CXR has high sensitivity $(87 \%-98 \%)$ but low specificity (46\%-89\%) for PTB, depending on the interpretative skills of the radiologist. ${ }^{(6)}$ Typical CXR manifestations of PTB are cavitation in the apical/apicoposterior segments of the upper lobes or the superior segment of the lower lobes. However, the radiological manifestations are mainly non-specific, mimicking other conditions such as malignancy and pneumonia. In settings with intermediate and high TB incidence, there should be a high index of suspicion for PTB in persons with abnormal CXR. Clinicians should be aware that radiology reports of scarring, fibrosis and granuloma, which may imply old, healed (i.e. inactive) TB, do 
not necessarily exclude active PTB, and that microbiological testing of sputum must be carried out. Clinicians should also be cognisant of the entity of culture-negative $\mathrm{PTB}^{(7)}$ and appreciate that treatment of patients with early disease has individual and public health benefits in preventing late disease manifestations and minimising transmission in the community.

Early detection and treatment of TB is of the highest priority in TB control. Delay in treatment not only leads to poorer patient outcomes, but also prolongs the exposure period of the infectious case in the community and is associated with greater transmission to contacts. ${ }^{(8)}$ Delayed TB diagnosis may be due to the patient or healthcare system. A systematic review by Storla et al of 58 studies addressing diagnostic delay found heterogeneous risk factors associated with the delay, which included: HIV; coexistence of chronic cough and/or other lung diseases; negative sputum smear; extrapulmonary TB; rural residence; low access (geographical or sociopsychological barriers); initial visitation of a government low-level healthcare facility, private practitioner, or traditional healer; old age; poverty; female gender; alcoholism and substance abuse; history of immigration; low educational level; low awareness of TB; incomprehensive beliefs; self-treatment; and stigma. The symptoms reported in 25 studies were cough $(85 \%)$, fever $(65 \%)$, weight loss $(62 \%)$, chest symptoms $(50 \%)$ and haemoptysis (25\%). Less frequently reported symptoms were sputum, fatigue and increased sweating. ${ }^{(9)}$ Although cough is the most common presentation of the disease, the pathophysiology of TB is such that patients may have low-grade, intermittent symptoms (which they may not bring to medical attention) yet are capable of transmitting the infection to their close contacts in the community.

One limitation of our study is that CXRs done in the private sector or sectors not linked to the EMR system were not captured. Thus, our findings do not fully reflect, and likely underestimate, the extent of missed opportunities for earlier diagnosis in patients who had previously abnormal CXRs prior to PTB diagnosis. Furthermore, we were unable to ascertain the circumstances behind 'no action taken' in response to the abnormal CXRs and can only speculate that one of the following occurred: (a) the abnormal CXR report was missed by the requesting physician; (b) the report was noted but not acted upon; or (c) the intention to act was not translated into concrete action. As most of the CXRs were performed for indications unrelated to TB, it is possible that attention may not have been paid to the abnormal CXR results in many of these cases. This is a worrying finding that has implications not only for missed early diagnosis of PTB, but also that of lung carcinoma.

Our study shows that within our public healthcare institutions, failure to recognise or take appropriate action in response to abnormal CXRs has resulted in diagnostic delay of TB for as long as several years. There is a need to increase vigilance and improve our healthcare system such that CXR findings are noted and acted upon in a timely and appropriate manner. In Singapore, a country with intermediate TB incidence, active PTB should be considered as a differential diagnosis in persons with abnormal CXR and sputum specimens sent for microbiological testing even in the absence of classical symptoms.

\section{REFERENCES}

1. World Health Organization. Global Tuberculosis Report 2018. Available at: https://www.who.int/tb/publications/global_report/en/. Accessed December 21, 2018

2. Ministry of Health, Singapore. Communicable disease surveillance in Singapore 2017. Available at: https://www.moh.gov.sg/resources-statistics/reports/ communicable-diseases-surveillance-in-singapore-2017. Accessed December 21, 2018.

3. Chee CB, Emmanuel SC, Wang YT. A brave STEP forward--the Singapore Tuberculosis Elimination Programme. Singapore Med J 1997; 38:359-60.

4. Chee CB, James L. The Singapore Tuberculosis Elimination Programme: the first five years. Bull World Health Organ 2003; 81:217-21.

5. World Health Organization. Systematic screening for active tuberculosis: principles and recommendations. Available at: https://www.who.int/tb/ tbscreening/en/. Accessed December 21, 2018.

6. Barry CE 3rd, Boshoff HI, Dartois V, et al. The spectrum of latent tuberculosis: rethinking the biology and intervention strategies. Nat Rev Microbiol 2009; 7:845-55.

7. Nahid P, Dorman SE, Alipanah N, et al. Official American Thoracic Society/ Centers for Disease Control and Prevention/Infectious Diseases Society of America Clinical Practice Guidelines: Treatment of Drug-Susceptible Tuberculosis. Clin Infect Dis 2016; 63:e147-95.

8. Golub JE, Bur S, Cronin WA, et al. Delayed tuberculosis diagnosis and tuberculosis transmission. Int J Tuberc Lung Dis 2006; 10:24-30.

9. Storla DG, Yimer S, Bjune GA. A systematic review of delay in the diagnosis and treatment of tuberculosis. BMC Public Health 2008, 8:15. 